\title{
Prompt but inefficient: nicotine differentially modulates discrete components of attention
}

\author{
Signe Vangkilde $\cdot$ Claus Bundesen • Jennifer T. Coull
}

Received: 24 March 2011 / Accepted: 16 May 2011 / Published online: 1 June 2011

(C) The Author(s) 2011. This article is published with open access at Springerlink.com

\begin{abstract}
Rationale Nicotine has been shown to improve both memory and attention when assessed through speeded motor responses. Since very few studies have assessed effects of nicotine on visual attention using measures that are uncontaminated by motoric effects, nicotine's attentional effects may, at least partially, be due to speeding of motor function.

Objectives Using an unspeeded, accuracy-based test, the CombiTVA paradigm, we examined whether nicotine enhances attention when it is measured independently of motor processing.

Methods We modelled data with a computational theory of visual attention (TVA; Bundesen 1990) so as to derive independent estimates of several distinct components of attention from performance of the single task: threshold of visual perception, perceptual processing speed, visual shortterm memory storage capacity and top-down controlled selectivity. Acute effects of nicotine (2 $\mathrm{mg}$ gum) on performance were assessed in 24 healthy young nonsmokers in a placebo-controlled counterbalanced, crossover design. Chronic effects of nicotine were investigated in 24 age- and education-matched minimally deprived smokers. Results Both an acute dose of nicotine in non-smokers and chronic nicotine use in temporarily abstaining smokers
\end{abstract}

S. Vangkilde $(\bowtie) \cdot$ C. Bundesen

Center for Visual Cognition, Department of Psychology,

University of Copenhagen,

Oester Farimagsgade 2A,

1353 Copenhagen K, Denmark

e-mail: Signe.Vangkilde@psy.ku.dk

J. T. Coull

Laboratoire de Neurobiologie de la Cognition,

Université de Provence \& CNRS,

Marseille, France improved perceptual thresholds but slowed subsequent perceptual speed. Moreover, both acute and chronic nicotine use reduced attentional selectivity though visual short-term memory capacity was unimpaired.

Conclusions Nicotine differentially affected discrete components of visual attention, with acute and chronic doses revealing identical patterns of performance. We challenge prior reports of nicotine-induced speeding of information processing by showing, for the first time, that nicotine slows down perceptual processing speed when assessed using accuracy-based measures of cognitive performance.

Keywords Nicotine $\cdot$ Attention $\cdot$ Cognition $\cdot$ Perceptual processing speed $\cdot$ Selectivity $\cdot$ Memory $\cdot$ Smoker .

Non-smoker· Human

\section{Introduction}

In the last few decades, drugs targeting cholinergic function have been suggested as therapeutic treatments for cognitive dysfunction in neurodegenerative and neuropsychiatric disorders, as well as potential cognitive enhancers in normal subjects (Everitt and Robbins 1997; Ferrea and Winterer 2009; Sarter and Parikh 2005). Nicotine acts as an agonist at nicotinic acetylcholine receptors (nAChRs) in the brain and modulates sensory responsiveness, psychomotor function and information processing in both humans and animals (Lawrence et al. 2002; Levin et al. 2006; Metherate 2004; Rezvani et al. 2002). Originally, cholinergic effects on cognition were thought to pertain to learning and memory, but this view was challenged by several experiments showing that cholinergic effects on mnemonic or learning processes vary as a function of attention (e.g., Rusted and Warburton 1992; for overviews, see Sarter et al. 
2003; Coull 2005). Specifically, it has been suggested that cholinergic activity in the brain is pivotal for certain aspects of attention and only determines the efficacy of learning and memory through its role in attentional processes (Sarter et al. 2003; Sarter and Parikh 2005).

The majority of early human studies showing a beneficial effect of nicotine on attention employed nicotinic manipulations in smokers (Sherwood 1993). However, in a review of 101 studies, Heishman et al. (1994) concluded that nicotine reliably enhanced only a few cognitive measures in non-smokers, among these finger tapping and motor responding in tests of attention. Furthermore, they concluded that both divided and selective attention are generally much less susceptible to nicotinic influences than tasks relying heavily on sustained attention. Sherwood (1993) reached a similar conclusion in his review, suggesting that nicotine may act as a mild CNS stimulant resulting in reduction in the vigilance decrement and general facilitation of motor responding, regardless of previous smoking experience. The latter idea was recently supported by a pharmaco-fMRI study (Rose et al. 2010) in which nicotinic effects on measures of attention were mediated not only by enhanced perceptual selectivity but also through facilitation of motor readiness. Following this line of reasoning, attentional effects ascribed to nicotine could in part be due to alterations in motor readiness, as many attentional tests employ motor-dependent response measures, such as simple reactions times or speeded accuracy.

In a recent meta-analysis of over 40 studies, including non-smokers and minimally deprived or non-deprived smokers, Heishman et al. (2010) divided studies into reaction time (RT) or accuracy-based outcomes and found beneficial nicotinic modulation of simple motor performance, alerting attention (accuracy and RT) and orienting attention (only RT, not accuracy). Furthermore, their results also reintroduced the possibility of a nicotinic involvement in memory, considering that both short-term episodic memory accuracy and working memory RT generally improved with nicotine. However, the latter effect could reflect a speed-accuracy trade-off, as five out of seven working memory tests reporting both types of measures showed a speeding of RTs accompanied by a decrease in accuracy. Heishman et al. claimed that the beneficial cognitive effects observed are likely to represent genuine performance enhancement. Nevertheless, they argued that to truly understand the effects of nicotine on human cognition, studies are needed that assess several aspects of human performance simultaneously and enable the modelling of complex performance in the laboratory. In parallel, Vossel et al. (2011) have called attention to the lack of studies comparing non-smokers and smokers, which are necessary for clarifying the cognitive effects of acute and chronic doses of nicotine, respectively.
Based on existing literature, we suggest that two outstanding questions have yet to be answered. First, does nicotine enhance the speed of attentional processing when estimated independently of motor processing? Although the effects of nicotine on speed-based measures of selective attention, such as the Posner covert orienting of attention task (e.g., Thiel and Fink 2008; Thiel et al. 2005), are unlikely to be adequately explained by modulation of motor processing, it is nevertheless necessary to test whether nicotine can also be shown to affect attentional speed in an entirely non-motor context. If so, we can more confidently refute the claim that nicotine's effects on RT-based attentional tasks are mere consequences of more fundamental effects on motor processing. Second, we ask whether nicotinic effects on discrete components of attention, such as processing speed, selectivity or visual short-term memory, can be dissociated when these are measured simultaneously within the same experimental paradigm? In addition, we use this opportunity to examine whether the attentional performance of non-smokers in an acute nicotinic challenge mimics the effects of chronic nicotine in minimally deprived smokers.

The TVA (Bundesen 1990) provides an ideal framework for investigating these questions. In contrast to most computerised attention tests using RT measures, TVAbased testing uses unspeeded, accuracy-based measures of attention unconfounded by motor components. TVA-based testing taps several attentional core functions such as how much material can be stored in visual short-term memory (VSTM) and how fast visual information can be processed. Furthermore, with TVA-based testing, it is possible to measure the threshold of conscious perception and the selectivity of attention, as well as to characterise how participants distribute their attention over space. In addition, in contrast to prior studies that have employed a number of diverse tasks to examine effects of nicotine on discrete components of attention, TVA allows for a more streamlined approach in which a number of perceptual, attentional and mnemonic measures are derived from performance of a single task. Crucially, this approach allows us to control for drug effects on potentially confounding processes of non-interest, such as motor responding or perceptual load, which, in traditional test batteries, often vary widely between tasks.

TVA is a formal computational theory that describes attention as a set of processes that allow us to select the information that is currently relevant for behavior (for a comprehensive account of these, see Bundesen 1990). According to TVA, the selection process can be described as a parallel processing race in which possible characterisations of the objects in our visual field compete for access to a VSTM with a limited capacity of $K$ elements (one of the basic parameters of the model). Only the winners of the 
race that are selected and encoded into VSTM become available for consciousness and action. However, the chances of winning the race are not equal for all objects and categories: In line with the ideas of Desimone and Duncan (1995), the race is seen as a biased competition, where not only the sensory evidence of certain categorisations but also attentional weights and subjective attentional biases govern the probabilities of encoding certain objects and categories. This encoding and selection process proceeds in two stages: During the first stage, attentional weights are calculated for every element in the visual field. Basically, the weight reflects the strength of the sensory evidence that the element is relevant. In the second stage, the total processing capacity of the visual system is distributed to the elements in proportion to their attentional weights. The capacity allocated to a particular element determines how fast this element is processed and, thus, how likely it is to become encoded into VSTM. In TVA, the total processing capacity of the visual system is assumed to be a constant, independent of the number of elements in the stimulus display (provided that the elements are homogenous in the sense of Bundesen 1990, p. 524). The value of this constant, $C$ elements per second, is also a basic parameter of the model.

TVA has successfully accounted for a wide range of behavioural and neurophysiological attentional effects (for an overview, see Bundesen and Habekost 2008). As such, TVA provides a general theoretical framework for investigating and explaining attention in normal subjects (Finke et al. 2005; Jensen et al. 2011) and in patients (Bublak et al. 2005, 2006; Duncan et al. 1999; Habekost and Rostrup 2006; 2007; Redel et al. 2011), as well as for explaining the effects of psychopharmacological manipulation (Finke et al. 2010).

\section{Method}

\section{Participants}

For the study 24 non-smoking participants (20 women, four men, $M_{\mathrm{age}}=23$ years, $\mathrm{SD}=2.3$ years) and 24 regularly smoking participants ( 17 women, seven men, $M_{\text {age }}=24.6$ years, $\mathrm{SD}=2.5$ years) were recruited at the Faculty of Social Sciences at the University of Copenhagen. To avoid confounding effects from previous nicotine dependence in the non-smoker group, we only recruited participants that reported never to have been regular smokers nor smoked within the last 2 years. Conversely, all participants in the smoker group were required to have been smoking regularly for at least the last 2 years. All participants but one (nonsmoker) were right-handed (Oldfield 1971), had normal or corrected-to-normal vision and no prior history of neurological or psychiatric disease. All participants reported to be unmedicated except for contraceptives and, in one case, insulin. We chose to include the data from this non-smoking subject, as this did not change the results. However, the results of two of the smokers are not reported, as it turned out after testing that these participants satisfied the exclusion criteria (specifically, colour blindness and antidepressant medication).

Both participant groups were asked to abstain from alcohol for at least $12 \mathrm{~h}$ before testing and from caffeine for at least $3 \mathrm{~h}$ before testing. The non-smokers participated in two sessions separated by 1 week (see below) and received 80 Euros as a financial compensation for their involvement in the study. The smokers were tested only once and received 40 Euros for their participation. The study was carried out in accordance with the ethical principles of the World Medical Association (Declaration of Helsinki) and was approved by the national ethical research committee (reference number H-D-2008-092). Written informed consent was obtained for all participants before entering the study.

\section{Procedure}

Drug administration and testing of non-smokers Drug administration in the non-smokers was double-blinded, randomised and counter-balanced. To enhance the sensitivity of the study, we employed a within-subject design, and thus, the non-smokers received either a single oral dose of nicotine in a polacrilex gum (Nicotinell $2 \mathrm{mg}$, Novartis Healthcare A/ S) or a placebo gum with matched taste (Novartis Healthcare A/S) in two separate sessions. Specifically, 12 participants received placebo in their first session and nicotine in the second, while the remaining participants received the treatments in the reverse order. Sessions were separated by at least 1 week to allow for complete wash-out of any nicotine received in the first session. Participants were instructed to chew the gum continuously for $1 \mathrm{~min}$ and then let it rest for 2-3 min, repeated over $30 \mathrm{~min}$ during which they remained seated in a designated waiting area. Nicotine from nicotine gum is absorbed through the oral mucosa throughout the period of chewing. During this period, plasma nicotine concentrations increase slowly, reaching a plateau after $30 \mathrm{~min}$ and then slowly decline over the next $2 \mathrm{~h}$ (Benowitz et al. 1988). Choi et al. (2003) investigated the pharmacokinetics of 2-mg nicotine gum and found a maximal plasma concentration after $0.8 \mathrm{~h}(\mathrm{SD}=0.2 \mathrm{~h})$ and a half-life of $2.5 \mathrm{~h}(\mathrm{SD}=1.2 \mathrm{~h})$. Even with considerable variability in individual pharmacokinetic responses, we assume that the non-smokers had all reached their maximal plasma concentration after the 30 -min pretest phase and showed only minor changes in nicotine concentration during the 45-min test session. 
Non-smoker subjective effect ratings To assess the subjective effects of nicotine, the non-smokers were asked to complete a set of visual analog scales (VAS, see Bond and Lader 1974) on three occasions during each session. The VAS had the words "not at all" and "extremely" at each end of a 10-cm line where participants were told to report their answer to six different questions by marking a point on the line corresponding to their subjective experience. Questions were chosen to reflect effects of fatigue due to testing and the most common complaints of adverse effects of nicotine in non-smokers. The questions were: "How sleepy do you feel right now?", "How dizzy do you feel right now?", "How nauseous do you feel right now?", "How blurred is your vision right now?", "How dry is your mouth right now?" and "How alert do you feel right now?" The scales were presented at baseline (just prior to drug administration), $30 \mathrm{~min}$ after drug administration (pretest) and $90 \mathrm{~min}$ after drug administration (posttest).

Smoking history and behaviour Smokers were tested in one session only and before completing the attention test (see below), they answered three questions about their smoking behaviour: "How many years have you been smoking?", "How many cigarettes do you smoke a day on average?" and "How many hours is it since your last cigarette?" The smokers had been informed that they were allowed to smoke up to $2 \mathrm{~h}$ before the testing session but not during the test. This procedure was chosen to avoid both withdrawal effects and acute effects of nicotine.

\section{Experimental paradigm}

The CombiTVA paradigm was designed specifically for this study as a combination of two classical experimental paradigms: the whole report paradigm (Sperling 1960) where all letter stimuli must be reported, and the partial report paradigm (see Shibuya and Bundesen 1988) in which only stimuli with a certain target feature (e.g. colour) are to be reported. The CombiTVA test took $45 \mathrm{~min}$ to complete and comprised 24 practice trials and nine experimental blocks of 36 trials. All trials followed the same basic design outlined in Fig. 1. A trial was initiated by a red fixation cross in the middle of a black screen. This was succeeded by a $100-\mathrm{ms}$ blank screen before the stimulus display was presented on an imaginary circle $\left(r=7.5^{\circ}\right.$ of visual angle) around the fixation cross with six possible stimulus locations. After a variable stimulus duration, the letter display was terminated by a 500 -ms masking display containing six masks made from red and blue letter fragments. Then, the screen turned black, and the participant could type in the letters that he or she had seen. In whole report trials, either two or six red target letters were presented, while partial report trials featured two red target letters and four blue distractor letters. Displays with six target letters were shown for each of six stimulus durations $(10,20$, $50,80,140$ or $200 \mathrm{~ms}$ ), while all other displays were shown for $80 \mathrm{~ms}$. All trial types were intermixed, and the stimuli in a given trial were chosen randomly without replacement from a set of 20 capital letters (ABDEFGHJKLMNOPRSTVXZ) written in the font Ariel (broad) with a letter point size of 68 corresponding to $2.7^{\circ} \times 2.3^{\circ}$ of visual angle. The individual, multicoloured masks were $100 \times 100$ pixels to completely cover the letters.

Participants were instructed to make an unspeeded report of all red letters they were "fairly certain" of having seen, that is, to use all available information but refrain from pure guessing. Participants were informed of the accuracy of their reports (the probability that a reported letter was correct) after each block, and they were encouraged to report as many red letters as possible but keep their reports within a specified accuracy range of $80-90 \%$ correct. The stimulus displays were presented on a 19 " CRT monitor at $100 \mathrm{~Hz}$ using the E-prime 2 software. All tests were run in a semidarkened room, with participants seated approximately $60 \mathrm{~cm}$ from the monitor.

\section{Estimation of TVA parameters}

The number of correctly reported letters in each trial constituted the main dependent variable in the CombiTVA test. The performance of the participants across the different test conditions was modelled by TVA using a maximum likelihood fitting procedure (for details, see Kyllingsbæk 2006 and Dyrholm et al. 2011). Through this procedure, five parameters quantifying attentional functions were estimated: (1) $K$, the capacity of visual short term memory measured in number of letters; (2) $C$, the speed of visual processing measured in letters processed per second; (3) $t_{0}$, the threshold of conscious perception or the longest ineffective exposure duration measured in seconds; (4) $\alpha$, the top-down controlled selectivity defined as the ratio between the attentional weight of a distractor and the attentional weight of a target, so that $\alpha$ values close to 0 indicate efficient selection of targets and values close to 1 indicate no prioritising of targets compared with distractors; and finally (5) $w_{\text {index }}$, the spatial distribution of attentional weighting defined as the ratio between the sum of attentional weights assigned to elements in the left hemifield and the sum of attentional weights across the entire visual field in the partial report trials. In this laterality index, $w_{\text {index }}$, a value of 0.5 indicates unbiased spatial weighting of attention, whereas values closer to 0 reflect a right-sided bias and values approaching 1 reflect that more attentional resources are used on the left side of the visual field.

The relationship between the first three parameters can be visualised by plotting the mean number of letters correctly 
Fig. 1 Outline of a single trial in the CombiTVA paradigm showing timing and three types of letter displays used: six target whole report (red letters), two target whole report (red letters), and two target and four distractor partial report (red and blue letters)

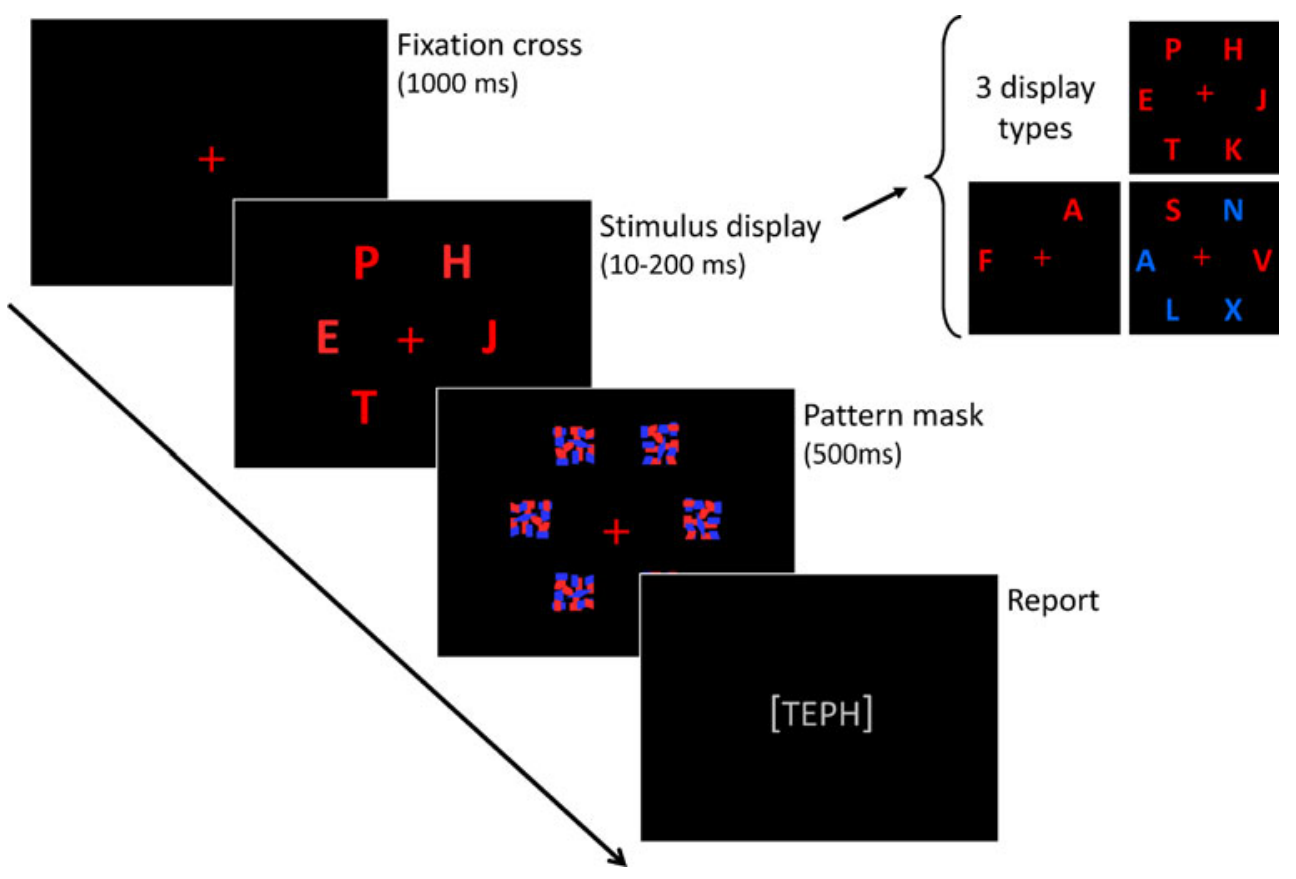

reported at each of the six different stimulus durations from the six-target whole report part of the CombiTVA test. Figure 2, upper panel, shows the observed performance of a single, representative subject and the predicted performance following an exponential curve based on the parameter estimates obtained through the TVA-based fitting procedure. The subject does not report anything at the shortest stimulus duration of $10 \mathrm{~ms}$, but the mean
Fig. 2 Upper panel Whole report performance of a representative non-smoker in the placebo condition (PLA) showing the mean number of correctly reported letters as a function of exposure duration. The solid curve represents a TVA-based fit to the observations. The estimated visual short-term memory capacity, $K$, is the horizontal asymptote of the fitted curve. $t_{0}$ is the longest ineffective exposure duration, the point at which the curve rises from the abscissa. The slope of the curve at $t_{0}$ corresponds to the perceptual processing speed, C. Lower panel Nicotine induced changes in whole report performance showing observed and estimated performance following placebo (PLA, diamonds and solid line) and observed and estimated performance following nicotine (NIC, dots and dashed line)
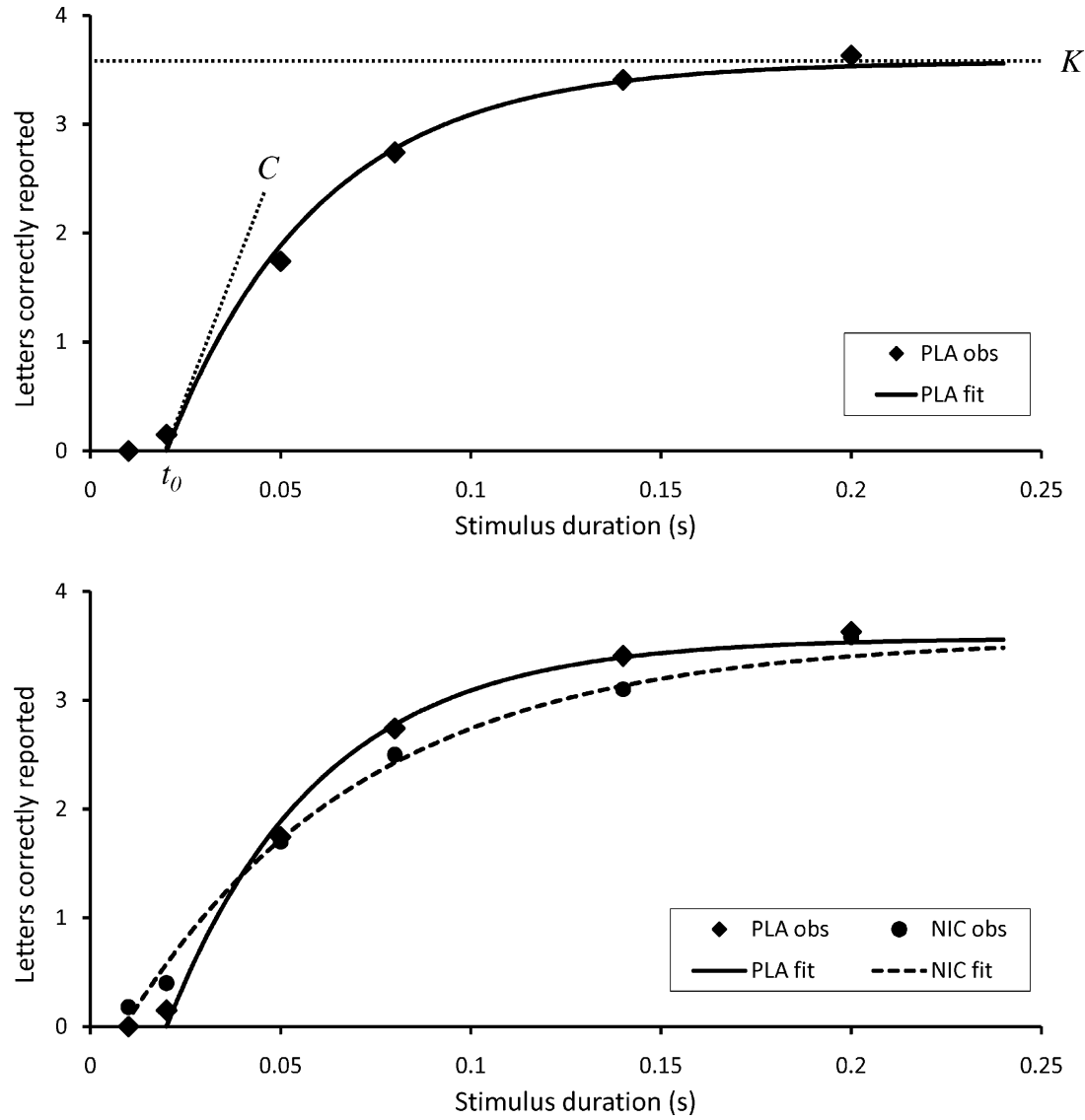
number of letters correctly reported rises steeply for the following durations and levels off at approximately 3.5 letters at the longer exposures. The point at which the curve rises from the abscissa represents the $t_{0}$ value: At exposures briefer than $t_{0}$, nothing can be reported. The slope of the curve at $t_{0}$ represents the $C$ value: The steeper the slope, the more letters can be processed per second. Finally, the horizontal asymptote of the curve is the maximum number of items that can be retained in visual short-term memory: the $K$ value.

The efficiency of top-down selection, represented by the $\alpha$ value, is estimated by comparing performance in the partial report trials (two targets and four distractors presented simultaneously) with performance in the whole report trials (two targets presented alone). A participant with perfect selection should be unaffected by the presence of distractors and thus report the same number of targets regardless of the number of distractors.

\section{Results}

Statistical analyses were carried out using PASW statistics version 18. For all analyses, differences were considered to be statistically significant at the 0.05 level. Effect measures for $t$ tests and analyses of variance are stated as $\eta_{\mathrm{p}}^{2}$ and Cohen's $d$, respectively. For repeated measures, the $d$ value was corrected for correlations between samples (see Dunlap et al. 1996). Finally, several of the attentional parameters measured here have been shown to be negatively affected by age; therefore, interparameter correlations were calculated as partial correlations assuming a linear effect of age (Field 2009). This did not change the conclusions in any way.

Attention in non-smokers and smokers

The parameter estimates obtained through the TVA-based fitting procedure correspond to values previously found in groups with comparable age and education (Jensen et al. 2011). The mean values for each of the three groups (nonsmokers in placebo and nicotine conditions, and smokers) are summarised in Table 1. On average, the smokers had the lowest threshold of conscious perception, $t_{0}$, followed by non-smokers under nicotine and non-smokers under placebo. Conversely, the fastest mean processing speed, $C$, was observed in non-smokers under placebo, followed by non-smokers under nicotine and finally smokers. The same pattern was repeated for selectivity, where non-smokers under placebo were the most selective and smokers the most distractible. There was, however, large variation in all three groups. The mean capacity of visual short term memory, $K$, was nearly identical in all groups, as was the
Table 1 Attentional performance of non-smokers and smokers

\begin{tabular}{lccc}
\hline Parameter & \multicolumn{2}{l}{ Non-smokers } & \multirow{2}{*}{ Smokers } \\
\cline { 2 - 3 } & $\begin{array}{l}\text { Placebo } \\
\text { M (SD) }\end{array}$ & $\begin{array}{l}\text { Nicotine } \\
M(\mathrm{SD})\end{array}$ & $M(\mathrm{SD})$ \\
\hline$t_{0}$ & $15(8)$ & $12(8)$ & $10(9)$ \\
$C$ & $70(24)$ & $55(23)$ & $51(18)$ \\
$K$ & $3.30(0.50)$ & $3.20(0.60)$ & $3.20(0.70)$ \\
$\alpha$ & $0.53(0.26)$ & $0.64(0.37)$ & $0.68(0.27)$ \\
$w_{\text {index }}$ & $0.47(0.08)$ & $0.50(0.08)$ & $0.49(0.09)$ \\
Error rate & $0.18(0.05)$ & $0.20(0.07)$ & $0.24(0.09)$ \\
\hline
\end{tabular}

Units for the individual parameters are $t_{0}(\mathrm{~ms}), C$ (letters/second), $K$ (letters), $\alpha$ ranges from perfect selection at 0 to non-selectivity at 1 , $w_{\text {index }}$ ranges from complete rightward bias at 0 to complete leftward bias at 1 with 0.5 indicating equal weighting between the two visual fields

spatial distribution of attention (as measured by $w_{\text {index }}$ ). The non-smokers in the placebo condition did show a small rightward bias in spatial attention, but even though the laterality index approached a significant deviation from the optimum value of 0.5 , the size of this effect was only moderate, $t(23)=-2.02, p=0.06, d=0.58$. Attentional weighting of the smokers and non-smokers under nicotine did not deviate from the unbiased $w_{\text {index }}$ of 0.5 , both $t \mathbf{s}<1$.

The error proneness of participants was estimated as the probability that a reported letter was incorrect (i.e. a distractor or a letter not included in the display). On average, $18 \%$ of the reports made by non-smokers in the placebo condition were erroneous, rising to $20 \%$ in the nicotine condition. Nonsmokers were therefore within the instructed accuracy range (80-90\% correct reports). By contrast, $24 \%$ of the letters reported by the smokers were erroneous, meaning that they were actually less accurate than instructed.

The two capacity parameters, $C$ and $K$, have previously been shown to be moderately positively correlated in normal samples, reflecting a tendency for faster processing in subjects with large capacities of visual short-term memory (Finke et al. 2005). Our findings replicated this relationship in all three conditions: non-smokers under placebo, $r(21)=0.68, p<0.001$; non-smokers under nicotine, $r(21)=0.58, p=0.004$; and smokers, $r(19)=0.57, p=$ 0.007 , all corrected for age. No other inter-parameter correlations were significant for the non-smokers in either condition (all $p \mathrm{~s}>0.12$ ), whereas the perceptual threshold of the smokers was lowered as their average error rate increased, $r(19)=-0.62, p=0.003$, corrected for age.

Nicotine-induced attentional change in non-smokers

We analysed nicotine-induced changes in non-smokers by comparing placebo and nicotine sessions in mixed 
ANOVAs with drug (nicotine, placebo) as a within subjects factor and drug order (nicotine-placebo, placebo-nicotine) as a between subjects factor. Nicotine significantly improved the threshold of conscious perception by lowering $t_{0}, F(1,22)=4.83, p=0.04, \eta_{\mathrm{p}}^{2}=0.18$, thus advancing the point in time at which stimulus encoding began (Fig. 3, upper right panel). However, nicotine also severely slowed down subsequent visual processing speed, $C, F(1,22)=$ $31.42, p<0.001, \eta_{\mathrm{p}}^{2}=0.59$ (Fig. 3, upper left panel), and also compromised visual top-down selectivity, $\alpha, F(1,22)=7.01$, $p=0.02, \eta_{\mathrm{p}}^{2}=0.24$ (Fig. 3, lower left panel). The capacity of visual short term memory, $K$, was unaffected by the nicotinic challenge, $F(1,22)=1.57, p=0.22, \eta_{\mathrm{p}}^{2}=0.07$ (Fig. 3, lower right panel). Figure 2, lower panel, illustrates nicotinic effects on the $t_{0}, C$ and $K$ parameters derived from whole report performance for a single representative non-smoker. Nicotine also resulted in a small but significant leftward shift in spatial bias, $w_{\text {index }}, F(1,22)=9.56, p=0.01, \eta_{\mathrm{p}}^{2}=0.30$ and made subjects more error prone, $F(1,22)=5.50, p=0.03$, $\eta_{\mathrm{p}}^{2}=0.20$. Importantly, the order of the sessions in which the participants received placebo and nicotine did not affect any of the measures, as all main effects of drug order and interactions between drug and drug order were non-significant, all $F_{\mathrm{s}}<1.45$, all $p \mathrm{~s}>0.24$.

The correlations between nicotine and placebo conditions were moderate to large for all parameters and highly significant; thus, the reported changes appear to be very systematic: $K, r(21)=0.75, p<0.001 ; C, r(21)=0.84, p<$ $0.001 ; t_{0}, r(21)=0.57, p<0.01 ; \alpha, r(21)=0.81, p<0.001$; $w_{\text {index }}, r(21)=0.77, p<0.001$; error rate, $r(21)=0.64, p=$ 0.001 , all corrected for age.

The possibility exists that the increased error proneness observed after nicotine could somehow have influenced the estimates for the attentional parameters affected by nicotine. To control for this, we calculated the difference scores (i.e. score in placebo condition minus score in nicotine
Fig. 3 Mean TVA parameter estimates for smokers and for non-smokers following placebo and nicotine, respectively. Panels represent performance on four basic components of visual attention. Upper left panel C, the speed of visual processing; upper right panel, $t_{0}$, the threshold of conscious perception; Lower left panel $\alpha$, the top-down controlled selectivity where $\alpha$ values close to 0 reflect efficient selection and values close to 1 reflect non-selectivity; lower right panel $K$, the capacity of visual short term memory. Differences between the groups are indicated by $p$ values. Error bars show standard errors of the means
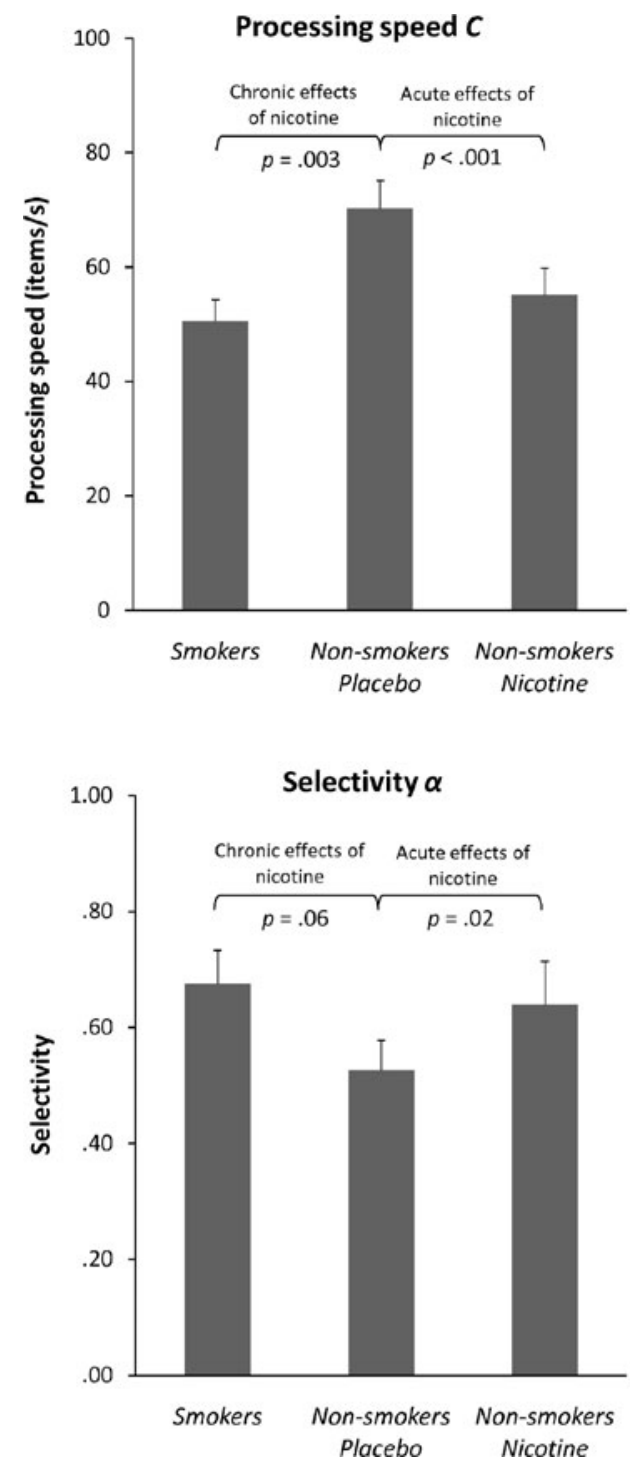
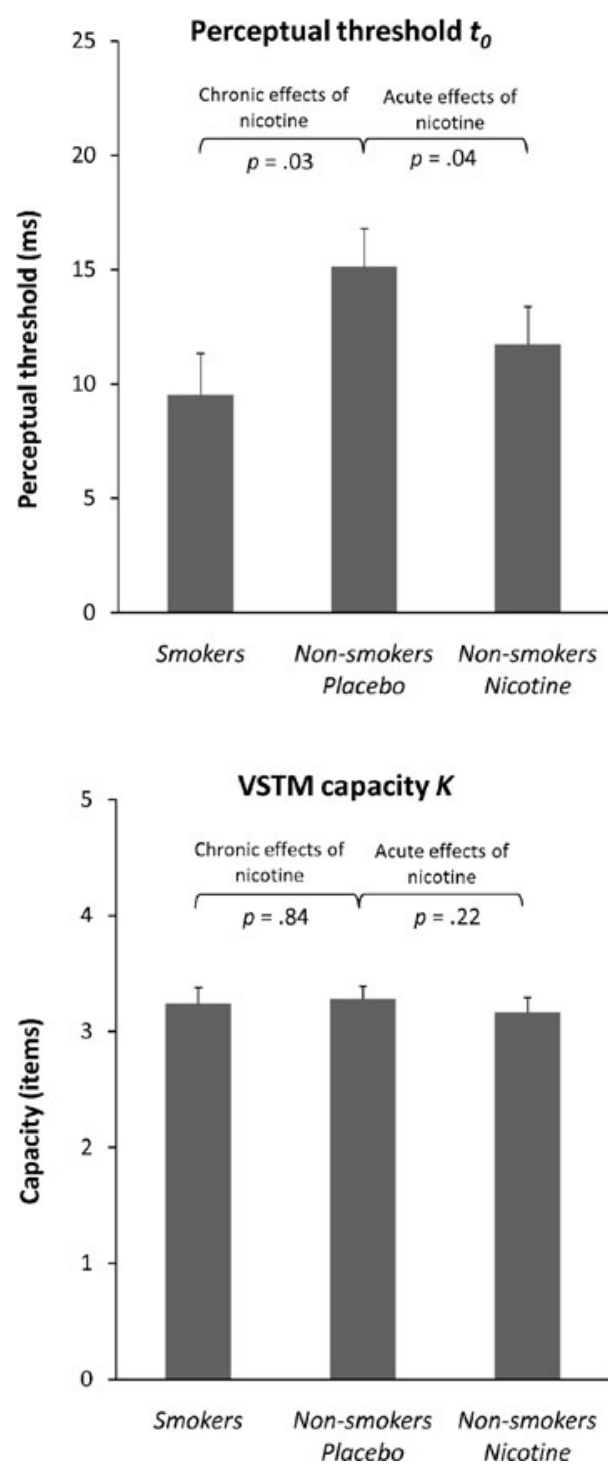
condition) for each parameter and correlated these to the difference score for the error rate. Correlations between error rate and TVA parameters were, on average, 0.13 (range, 0.01-0.28), and none were significant. Therefore, changes in error rate did not seem to influence nicotinic effects on attention parameters.

Finally, to investigate whether baseline performance was influencing the drug induced changes observed, we divided participants into two groups based on their placebo score (i. e. above or below the median placebo score) and used the resulting binary variable as a between subjects factor in a mixed measures ANOVA with drug (nicotine and placebo) as within subjects factor. We repeated this procedure for all five TVA-based parameters and found that none of the resulting interaction effects between drug and placebo score group were significant: $K, F(1,22)=3.56, p=0.07, \eta_{\mathrm{p}}^{2}=$ $0.15 ; C, F(1,22)=2.04, p=0.17, \eta_{\mathrm{p}}^{2}=0.09 ; t_{0}, F(1,22)=$ $2.28, p=0.15, \eta_{\mathrm{p}}^{2}=0.09 ; \alpha, F(1,22)=0.03, p=0.87, \eta_{\mathrm{p}}^{2}=$ $0.001 ; w_{\text {index }}, F(1,22)=1.02, p=0.32, \eta_{\mathrm{p}}^{2}=0.05$.

\section{Subjective measures in non-smokers}

As expected, none of the VAS measures differed significantly between the placebo and nicotine conditions at baseline (all $p \mathrm{~s}>0.16$ in paired $t$ tests), whereas several significant differences were found at pretest and posttest (see Fig. 4). The effects of nicotine and time-on-task were assessed in $2 \times 3 \times 2$ mixed measures ANOVAs with drug (nicotine and placebo) and time (baseline, pretest and posttest) as within subjects factors and drug order (nicotine-placebo and placebo-nicotine) as between subjects factor. All measures but the blurred vision measure were negatively affected by nicotine: sleepiness, $F(1,18)=$
6.82, $p=0.02, \eta_{\mathrm{p}}^{2}=0.28$; dizziness, $F(1,18)=13.33, p=$ $0.002 ; \eta_{\mathrm{p}}^{2}=0.43$; nausea, $F(1,18)=5.41, p=0.03, \eta_{\mathrm{p}}^{2}=0.23$; dry mouth, $F(1,18)=5.08, p=0.04, \eta_{\mathrm{p}}^{2}=0.22$; alertness, $F$ $(1,18)=6.30, p=0.02, \eta_{\mathrm{p}}^{2}=0.26$. This pattern repeated for the main effects of time where all measures but dry mouth were negatively affected by time-on-task: sleepiness, $F$ $(1.31,23.58)=10.59, p=0.002, \eta_{\mathrm{p}}^{2}=0.37 ;$ dizziness, $F$ $(1.28,23.03)=14.11, p<0.001, \eta_{\mathrm{p}}^{2}=0.44$; nausea, $F(2$, $36)=4.29, p=0.02, \eta_{\mathrm{p}}^{2}=0.19$; blurred vision, $F(1.36$, $24.38)=6.16, p=0.01, \eta_{\mathrm{p}}^{2}=0.26$; alertness, $F(1.27,23.16)=$ $10.45, p=0.002, \eta_{\mathrm{p}}^{2}=0.37$. The non-integer degrees of freedom reflect Greenhouse-Geisser corrections due to violations of assumed sphericity in the time factor. The effect of testing time was mainly driven by the changes from baseline in the nicotine condition (cf. Fig. 4), resulting in significant drug $\times$ time interactions for experience of dizziness $\left[F(2,36)=14.25, p<0.001, \eta_{\mathrm{p}}^{2}=0.44\right]$, dry mouth $\left[F(2,36)=8.58, p=0.001, \eta_{\mathrm{p}}^{2}=0.33\right]$ and alertness $[F(2$, $\left.36)=3.30, p=0.048, \eta_{\mathrm{p}}^{2}=0.16\right]$. Furthermore, there was a marginally significant interaction effect for nausea $[F(2$, $\left.36)=3.27, p=0.05, \eta_{\mathrm{p}}^{2}=0.15\right]$, but none for sleepiness or blurred vision (both $p \mathrm{~s}>0.14$ ). Again, the order in which the participants received the drugs did not affect any of the measures, all $F_{\mathrm{s}}<2.17$, all $p \mathrm{~s}>0.16$.

The relationship between the objective effects of nicotine and the participants' subjective experience of nicotine was explored by comparing the difference scores for the TVA parameters with difference scores for the VAS measures (placebo minus nicotine) at pretest and posttest, respectively. Despite the significant effects of nicotine on the subjective measures, they did not significantly correlate with the TVA parameters, at neither pre- nor posttest (average correlation $=0.14$; range, $0.001-0.40$ ).
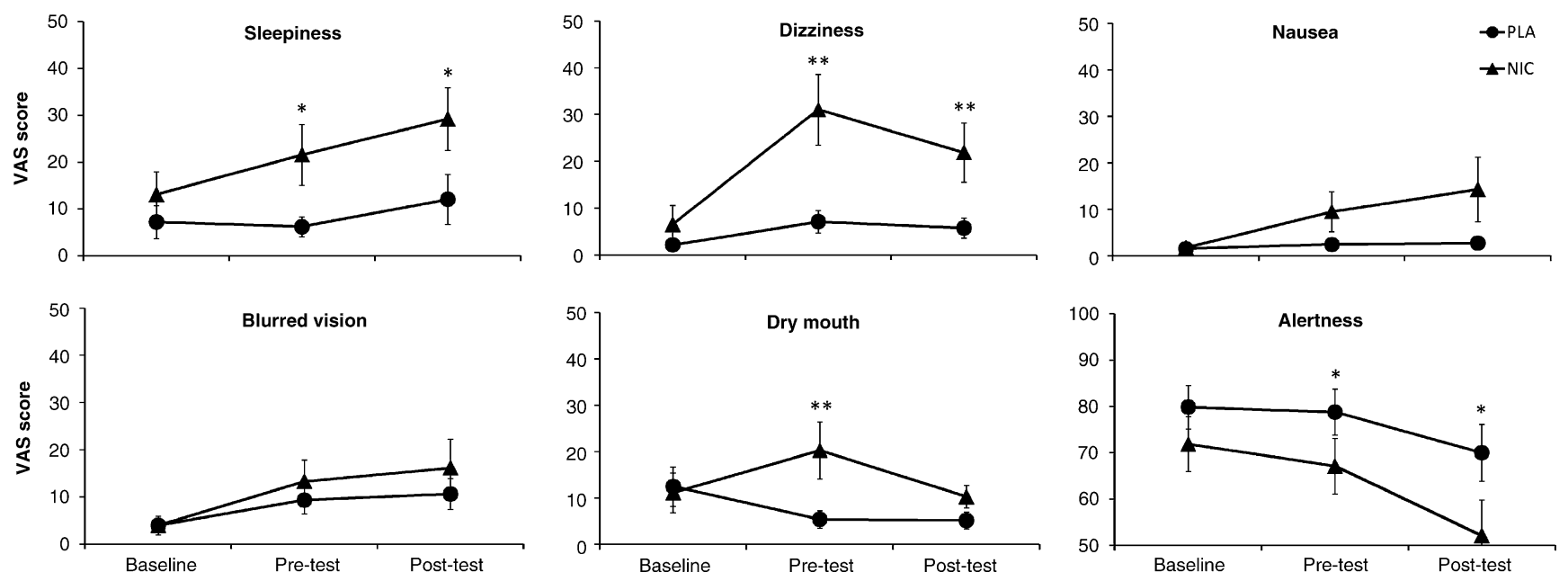

Fig. 4 Mean subjective effects of an acute dose of nicotine (triangles) or placebo (circles) in non-smokers. Data were collected using visual analog scales at baseline (just prior to drug administration), pretest (30 min after drug administration), and posttest (90 min after drug

administration). Statistically significant differences between drug conditions are indicated for $* p<0.05$ and $* * p<0.01$. Error bars show standard errors of the means 
Smoking behaviour in smokers

On average, the participants reported to have been smoking for 8.7 years $(\mathrm{SD}=3.25$ years) and to smoke on average $12.7(\mathrm{SD}=6.61)$ cigarettes a day. The median number of hours since their last cigarette at the time of testing was 2.75. One participant had not smoked for $72 \mathrm{~h}$ before testing, but the range for the remaining participants was $2-$ $15 \mathrm{~h}$. As the performance of the aforementioned participant did not deviate significantly from the rest of the subject group, her results were included in all analyses. As we expected, correlations showed that older participants had been smoking for more years, $r(20)=0.65, p=0.001$, and that participants who smoked fewer cigarettes per day had been abstinent for more hours before the test, $r(20)=0.46$, $p=0.03$. However, smoking behaviour was not correlated with any of the attentional parameters.

\section{Attention in non-smokers versus smokers}

The similarity of the smokers' performance to the nonsmokers under placebo and nicotine, respectively, was investigated in planned, independent $t$ tests with group (smoker/non-smoker) as a between-subjects factor. Compared to the non-smokers at placebo, the smokers had a significantly lower perceptual threshold, $t(44)=2.27, p=0.03, d=0.48$ (Fig. 3, upper right panel), and they showed a marked slowing of processing speed, $t(44)=3.14, p=0.003, d=0.67$ (Fig. 3, upper left panel). Similarly, the smokers made more errors, $t(30.90)=-2.67, p=0.01, d=-0.57$ (degrees of freedom corrected for unequal variances), and had a marginally significantly compromised selectivity, $t(44)=-1.92, p=0.06$, $d=-0.41$ (Fig. 3, lower left panel). Finally, neither the capacity of short term memory (Fig. 3, lower right panel) nor the spatial distribution of attention in the smokers were different from the non-smokers under placebo, both $t \mathbf{s}<1$, both $d \mathbf{s}<0.16$. By contrast, the performance of smokers was not significantly different from performance of non-smokers under nicotine, for any of the attentional parameters, all $t \mathbf{s}<1$, all $d \mathbf{s}<0.20$ (Fig. 3). This was also the case when comparing the error rates, $t(44)=2.16, p=0.15, d=-0.31$.

\section{Discussion}

In our study of cholinergic modulation of visual attention, we challenge the notion of the cholinergic agonist nicotine as a performance-enhancing drug. We examined the effects of both acute and chronic nicotine on visual attention using the new CombiTVA paradigm, which was designed to assess distinct components of visual attention within a single task. Acute nicotine administration in non-smokers resulted in a lower perceptual threshold, thus advancing the point in time at which encoding of information into visual short-term memory was begun. However, nicotine induced tradeoffs by slowing down subsequent information processing and weakening attentional selectivity, reflecting the fact that subjects were more prone to spend valuable resources on irrelevant information. Finally, nicotine had no effect on the capacity of visual short-term memory. Although nicotine increased subjective feelings of nausea, dizziness and dry mouth as well as decreasing alertness, these effects did not correlate with nicotine's effects on attentional performance. Consequently, the attentional effects were not driven by a lack of nicotine tolerance. The effects of chronic nicotine were characterised by comparing an age and education matched group of minimally deprived smokers to the non-smokers. Interestingly, the pattern of performance in the smokers closely matched the performance shown by non-smokers after an acute dose of nicotine: as compared to non-smokers receiving placebo, smokers also showed an earlier perceptual threshold, slower encoding into visual short-term memory and compromised selectivity.

Nicotine improves the perceptual threshold but slows subsequent perceptual processing speed

In our study, nicotine allowed stimuli to be perceived at significantly shorter exposure times, thereby lowering the perceptual threshold, $t_{0}$. Beneficial effects of nicotine on low-level perceptual processes have previously been found using both motor measures of performance, such as visual pop-out reaction time (Rycroft et al. 2005), or more perceptual measures, such as the critical flicker fusion threshold (Sherwood 1993). Similarly, another measure of perceptual sensitivity, the $d^{\prime}$ parameter, estimated through analyses of hits and false alarm rates by signal detection theory (Green and Swets 1966), has been shown to be enhanced by nicotine in near-threshold detection paradigms (Jones et al. 1992; Rezvani et al. 2002; Wesnes et al. 1983). Finally, several studies employing another accuracy-based paradigm, the visual inspection time (IT) task have shown beneficial effects of nicotine. IT is defined as the minimum stimulus exposure time required to reach near-perfect response accuracy in a simple two-choice visual discrimination and is argued to reflect stimulus input stages of visual perception (Stough et al. 1995). In abstaining (2$10 \mathrm{~h})$ smokers, nicotine improved early perception by lowering IT (Thompson et al. 2002), while the nicotinic antagonist mecamylamine prolonged IT in non-smokers (Thompson et al. 2000). This was proposed to be the result of a nicotinic improvement in perceptual processing speed. However, the IT procedure does not allow for separation of perceptual threshold from processing speed, which is a major strength of our CombiTVA paradigm. Our own 
results suggest more specifically that reduction in IT could be caused by nicotinic improvements in perceptual threshold, rather than processing speed per se. Taken as a whole, observations of lowered perceptual thresholds and improved perceptual sensitivity converge to support the notion that nicotine may enhance sensory responsiveness in the cortex (Metherate 2004; Sarter et al. 2005).

In contrast to the beneficial effects of nicotine on the perceptual threshold, both acute and chronic doses of nicotine slowed down visual information processing speed. However, the most well-established finding in the literature on effects of nicotine is a speeding of motor response time (Heishman et al. 1994, 2010; Sherwood 1993). Faster motor responses may not only reflect true enhancement of information processing but may also reflect speed-accuracy trade-off or purely motoric effects. Not all RT-based studies report accuracy outcomes but in the ones that do, faster RTs are often coupled with negative or null effects on accuracy (e.g. Thiel and Fink 2008). Correspondingly, in their recent meta-analysis Heishman et al. (2010) concluded that the enhancing effects of nicotine could be mediated through facilitation of motor processes. This view is supported by the neurobiology of the nicotinic system. Nicotinic receptors (nAChRs) play a substantial role in modulating synaptic transmission in several subcortical and cortical areas, including the striatum and parts of the motor cortex that are crucial for efficiency of motor responses (Feldman et al. 1997). In addition to the physiological effects on the CNS that presumably underlie changes in cognitive processes, nicotine also has peripheral physiological effects. In the doses normally applied in human studies, nicotine has an excitatory effect upon the nicotine cholinergic receptors at neuromuscular junctions (Feldman et al. 1997), and this could accentuate the possible central motor facilitation caused by nicotine. As our experiments did not include RT-based measures, we cannot determine whether our findings are part of a speed-accuracy trade-off induced by nicotine. However, this seems implausible given that subjects were explicitly instructed to give unspeeded reports. Rather, our results show that nicotinic effects on information processing speed are not exclusively beneficial. Thus, although numerous studies have previously found that nicotine enhances motor speed, we show for the first time that it slows down perceptual processing speed.

Nicotine impairs top-down attentional selectivity

We found that both acute and chronic nicotine impaired attentional selectivity. Nicotine effects on measures of selective attention are notoriously variable, with some studies reporting improvement while others show no effect (see Heishman et al. 1994, 2010 for reviews). Since many of the studies reporting beneficial effects of nicotine employed reaction time measures, Heishman et al. (2010) suggested that nicotine's effects on selectivity may in fact derive from motoric effects. In the present study, we have found that if attentional selectivity is measured with accuracy scores, nicotine actually impairs selectivity.

One of the tasks most commonly used to index selective attention is the spatial orienting paradigm (Posner et al. 1980), in which an initial cue directs attention to a spatial location in which a subsequent target may (valid trial), or may not (invalid trial), appear. The validity effect measures the behavioural cost (indexed by slowed reaction times) of detecting targets that appear in the non-cued location. Nicotine has consistently been found to reduce the validity effect, primarily by speeding responses to invalidly cued targets (Meinke et al. 2006; Phillips et al. 2000; Thiel and Fink 2008; Thiel et al. 2005; Vossel et al. 2008, 2011; Witte et al. 1997). Yu and Dayan (2005) suggested that the reduced validity effect after nicotine might reflect a less efficient use of the spatial information carried by the cue. This mechanism is entirely consistent with our own finding that nicotine impairs attentional selectivity: Nicotineinduced reduction in selectivity would result in a reduced reliance on the cue, and, consequently, invalidly cued targets would be processed more quickly following nicotine than following placebo.

Being less selective and more distractible may, however, in some cases confer a behavioural advantage. As previously discussed, performance in a cueing experiment, in which not being misled by invalid cues is important, might in fact benefit from a less focused use of the information provided by the cues. Likewise, being more distractible may also aid performance in prospective memory tasks, where participants are required to switch attention away from an attention demanding ongoing task in order to register and respond to an infrequently occurring prospective memory cue (Rusted et al. 2009; Rusted and Trawley 2006). Finally, there is evidence that the attentional blink, a transient impairment in the ability to report the second of two targets presented in close temporal succession, is attenuated if participants are actively required to distract their attention from the task at hand (see, e.g. Olivers and Nieuwenhuis 2006).

\section{Nicotine has no effect on VSTM capacity}

In our terminology, parameter $K$ is the storage capacity of VSTM, and VSTM can be considered synonymous with visual working memory, WM (cf. Baddeley 1999). The lack of nicotinic effect on $K$ fits well with the conclusions of a recent meta-analysis (Heishman et al. 2010) in which RTbased measures of WM were concluded to benefit from nicotine, whereas WM assessed through accuracy scores showed no significant change after nicotine. The null effect 
of nicotine on $K$ found in our study and several others (Ernst et al. 2001; Heishman and Henningfield 2000; Kleykamp et al. 2005; Myers et al. 2008) is supported by investigations demonstrating that WM and visuospatial attention performance have different genetic underpinnings (Greenwood et al. 2005; Parasuraman et al. 2005). Here attention - but not WM-was shown to be modulated by changes in a polymorphism on a gene crucial for one of the nAChRs. Conversely, the ability to retain several locations in WM-but not visuospatial attention-interacted with variation in a polymorphism on a gene involved in the conversion of dopamine to noradrenaline. Correspondingly, in a recent TVA-based study, modafinil, an analeptic drug increasing the release of dopamine and noradrenaline, was found to enhance visual short-term memory in lowperforming subjects (Finke et al. 2010). Thus, the WM aspect of human memory does not seem to depend on cholinergic nicotinic involvement, although prospective memory, the memory for an intention to perform a specific act, may do so (Heishman et al. 2010; Rusted et al. 2009; Rusted and Trawley 2006).

\section{Acute versus chronic effects of nicotine}

Using accuracy-based measures of attentional performance, we have found nicotine-induced impairments in the speed and selectivity of attentional operations. However, some prior studies have reported beneficial effects of nicotine, even when accuracy-based measures are used (Hahn et al. 2007; Jones et al. 1992; Lawrence et al. 2002). A possible interpretation of the discrepant findings was put forth by Newhouse et al. (2004), who pointed to differences in the populations represented in studies on nicotine: Studies showing null-effects or impairment after nicotine generally use non-smoking subjects, while studies using abstaining smokers or clinical populations tend to argue in favour of a beneficial effect of an acute dose of nicotine.

In our own study, we examined both acute attentional effects of nicotine in non-smokers and chronic attentional effects of nicotine in minimally deprived smokers, and found no difference between these effects. Importantly, we did not find any correlation between the smoking behaviour and history of the smokers and their attentional performance, even though the number of hours that the smokers had been abstaining could be expected to impact negatively upon performance. Comparisons of smokers to non-smokers in the absence of an acute nicotine challenge are relatively scarce (Vossel et al. 2011), but those that do exist demonstrate either impaired attentional performance for even mildly abstinent smokers (Foulds et al. 1996; Lawrence et al. 2002; Vossel et al. 2011) or else no difference between smokers and nonsmokers (Hahn et al. 2007, 2009; Rusted and Trawley 2006). Therefore, the acute and chronic effects of nicotine in smokers appear to counterbalance one another, which is of course consistent with the notion that acute nicotine in smokers serves to return the system to its "normal" functioning state. If attentional performance is related to nicotinic stimulation in a curvilinear relationship (i.e. as described by Yerkes and Dodson 1908), only an intermediate level of stimulation yields optimal performance, whereas low or high levels of stimulation will lead to impaired performance. As a consequence, for a normal nonsmoking individual performing optimally at baseline, acute nicotinic stimulation could have deleterious effects. Conversely, in both abstinent smokers and patients with reduced cholinergic tone due to neurocognitive disease who can be assumed to perform suboptimally at baseline, the same nicotinic stimulation could significantly enhance cognitive performance. In line with this, it has been suggested that the immediate relief from withdrawal symptoms offered by acute administration of nicotine in smokers or patients with a nicotine deficit plays a crucial role in nicotine addiction in those groups (Benowitz 2008).

One limitation of this study is that we did not examine the effects of an acute dose of nicotine in abstaining smokers. According to the observation of Newhouse et al., this should result in improvements in perceptual processing speed and selectivity, which would lie in contrast to the equivalent impairments we observed in non-smokers. Future studies will test this hypothesis using a placebocontrolled cross-over design to examine attentional effects in smokers, using different doses of nicotine in order to characterise a possible dose-dependent relationship between nicotinic stimulation and values of TVA-based attentional parameters. Furthermore, in order to further investigate the proposed dissociation between nicotinic effects on motor and perceptual processing speed, RT-based measures will be included in addition to purely accuracy-based measures.

Explaining nicotine effects with a theory of visual attention

The current neural interpretation of TVA (NTVA; Bundesen et al. 2005) does not account for the neurochemical basis of visual selection and recognition, but the theory does suggest some plausible neuroanatomical substrates for these processes. Specifically, the attentional weights computed for each stimulus object in the visual field are tentatively proposed to be stored in a saliency map in the pulvinar nucleus of the thalamus. Cortical processing capacity is then redistributed according to attentional weight signals from the pulvinar nucleus to the cortex, such that objects with high attentional weights are processed by more neurons than objects with low attentional weights. The encoding speed of an object is proportional not only to the number of neurons allocated to its processing but also to the level of activation of these neurons, which is governed by 
perceptual biases. These bias values are not generated within the visual system but derive from signals originating in frontal and parietal cortical areas.

The overall pattern of our findings may be explained in terms of NTVA by making two assumptions concerning the effects of nicotine. The first assumption is that nicotine truncated the computation of attentional weights, making it prompt but inaccurate. In TVA, $t_{0}$ is the longest ineffective exposure duration (see Fig. 2, upper panel), and this threshold depends on the time taken to compute attentional weights for objects presented in the visual field. Specifically, fast computations lead to lower temporal thresholds but imprecise weights, whereas elaborate computations result in higher temporal thresholds and more accurate weights. Visual selectivity, $\alpha$ (the ratio between the attentional weight of a distractor and the attentional weight of a target), depends critically on the precision of attentional weight estimates. Consequently, less accurate attentional weights result in an increment in the selectivity parameter, $\alpha$, indicating that valuable attentional resources are spent on irrelevant information. As touched upon previously with reference to spatial orienting paradigms, unfocused selectivity would result in differential speeding of processing invalid targets, whereas focused selectivity would favour processing of valid targets.

The second assumption is that nicotine caused a decrease in subjective biases for task-relevant (letter) categorisations, resulting in less efficient encoding of the letters into VSTM, evident in the slowing of the processing speed parameter, $C$. Note that although encoding of letters into VSTM should be slowed down, the number of items that can be stored in VSTM, $K$, should not be affected.

Support for these assumptions concerning nicotinic effects on attentional weights and perceptual biases comes from different areas of neuroscience. First, the pulvinar nucleus, which is likely to be critical for computing attentional weights, contains an abundance of presynaptic nicotinic receptors and receives substantial cholinergic projections from the pedunculopontine nucleus (Spurden et al. 1997). These projections are part of the reticularactivating system and probably play an important role in arousal and visual attention alike (Coull 1998). Second, Metherate (2004) reviewed the function of the nicotinic receptors (nAChRs) in the CNS and found an emerging consensus that cortical nAChRs likely act to change sensory responsiveness, which may contribute to improved behavioural performance in cognitive tasks. Metherate suggested that this is achieved by nicotinic regulation of glutamatergic and GABAergic activity in the thalamus, resulting in alterations in thalamo-cortical neurotransmission. Interestingly, Petersen et al. (1987) demonstrated the involvement of GABAergic activity in the pulvinar in a spatial cueing paradigm by injecting GABAergic drugs into parts of the pulvinar nuclei of rhesus monkeys. The procedure manipulated attentional performance in such a way that attentional shifts from one visual field to the other depended on the level of GABAergic activation in the contralateral pulvinar. Several researchers have interpreted this as support for the hypothesis that the pulvinar is crucial for the representation of attentional weights (Bundesen et al. 2005; Desimone and Duncan 1995). Finally, neuroimaging studies in humans have observed that thalamic and parietal regions are among the areas most consistently modulated by cholinergic or, more specifically, nicotinic, manipulations. Using PET, Mentis et al. (2001) found that nicotine specifically modulated activity in the thalamus and inferior parietal cortex, and this finding has been replicated many times since in pharmaco-fMRI studies, which show that nicotine (or acetylcholine in general) increases attention-related activity primarily in the pulvinar nucleus of the thalamus and in parietal cortex (Bentley et al. 2004; Hahn et al. 2007, 2009; Lawrence et al. 2002; Thiel et al. 2005 ). In summary, in an interpretation drawing upon NTVA and evidence from different areas of neuroscience, nicotinic modulation of thalamic areas might result in quickly computed but inaccurate attentional weights, whereas nicotinic modulation of parietal cortex might result in abnormal perceptual biases.

\section{Conclusion}

Both an acute dose of nicotine in non-smokers and chronic nicotine use in temporarily abstaining smokers diminishes perceptual thresholds while simultaneously slowing perceptual speed. In addition, both acute and chronic nicotine use reduce attentional selectivity but leaves short-term memory capacity unimpaired. Crucially, the nicotine-induced slowing in perceptual processes stands in contrast to prior reports of nicotine-induced speeding of motor processes.

Our results also serve to accentuate the utility of employing accuracy-based measures of cognitive performance in psychopharmacological studies, not least when investigating the effects of neurochemical substances that may also influence basic motor function. Moreover, using TVA to derive discrete measures of perceptual, attentional and mnemonic performance from the same, single task we could control for drug effects on potentially confounding processes of non-interest, such as perceptual load or motor function. Finally, the specificity of our TVA results attests to the use of TVA-based measures for psychopharmacological research, in which highly specific and sensitive measures of discrete components of attention are imperative. 
Acknowledgements This study was funded by grants from the University of Copenhagen Programme of Excellence (awarded to CB) and the European Collaborative Research Projects in the Social Sciences (awarded to JTC). SV was supported by a grant from the Nordic Center of Excellence in Cognitive Control (NCoE-CC). There is no conflict of interest pertaining to this study, which was carried out in accordance with current Danish laws concerning biomedical research and was approved by the national ethical research committee. We would like to thank Annemarie Hilkjær Petersen for assistance with testing the smokers, and Egill Rostrup for valuable medical advice.

Open Access This article is distributed under the terms of the Creative Commons Attribution Noncommercial License which permits any noncommercial use, distribution, and reproduction in any medium, provided the original author(s) and source are credited.

\section{References}

Baddeley AD (1999) Essentials of human memory. Psychology Press, Sussex

Benowitz NL (2008) Clinical pharmacology of nicotine: implications for understanding, preventing, and treating tobacco addiction. Clin Pharmacol Ther 83(4):531-541

Benowitz NL, Porchet H, Sheiner L, Jacob P (1988) Nicotine absorption and cardiovascular effects with smokeless tobacco use: comparison with cigarettes and nicotine gum. Clin Pharmacol Ther 44(1):23-28

Bentley P, Husain M, Dolan RJ (2004) Effects of cholinergic enhancement on visual stimulation, spatial attention, and spatial working memory. Neuron 41(6):969-982

Bond A, Lader M (1974) The use of analogue scales in rating subjective feelings. Br J Med Psychol 47:211-218

Bublak P, Finke K, Krummenacher J, Preger R, Kyllingsbaek S, Müller HJ et al (2005) Usability of a theory of visual attention (TVA) for parameter-based measurement of attention II: evidence from two patients with frontal or parietal damage. J Int Neuropsychol Soc 11(7):843-854

Bublak P, Redel P, Finke K (2006) Spatial and non-spatial attention deficits in neurodegenerative diseases: assessment based on Bundesen's theory of visual attention (TVA). Restor Neurol Neurosci 24(4-6):287-301

Bundesen C (1990) A theory of visual attention. Psychol Rev 97 (4):523-547

Bundesen C, Habekost T (2008) Principles of visual attention: linking mind and brain. Oxford University Press, Oxford

Bundesen C, Habekost T, Kyllingsbæk S (2005) A neural theory of visual attention: bridging cognition and neurophysiology. Psychol Rev 112(2):291-328

Choi J, Dresler C, Norton M, Strahs K (2003) Pharmacokinetics of a nicotine polacrilex lozenge. Nicotine Tob Res 5(5):635-644

Coull JT (1998) Neural correlates of attention and arousal: insights from electrophysiology, functional neuroimaging and psychopharmacology. Prog Neurobiol 55(4):343-361

Coull JT (2005) Psychopharmacology of human attention. In: Itti L, Rees G, Tsotsos JK (eds) Neurobiology of attention, vol 33. Elsevier Academic, London, pp 50-56

Desimone R, Duncan J (1995) Neural mechanisms of selective visual attention. Annu Rev Neurosci 18:193-222

Duncan J, Bundesen C, Olson A, Humphreys G, Chavda S, Shibuya H (1999) Systematic analysis of deficits in visual attention. J Exp Psychol Gen 128(4):450-478

Dunlap WP, Cortina JM, Vaslow JB, Burke MJ (1996) Meta-analysis of experiments with matched groups or repeated measures designs. Psychol Meth 1(2):170-177
Dyrholm M, Kyllingsbæk S, Espeseth T, Bundesen C (2011) Generalizing parametric models by introducing trial-by-trial parameter variability: the case of TVA (in press)

Ernst M, Matochik JA, Heishman SJ, Van Horn JD, Jons PH, Henningfield JE et al (2001) Effect of nicotine on brain activation during performance of a working memory task. PNAS 98 (8):4728-4733

Everitt BJ, Robbins TW (1997) Central cholinergic systems and cognition. Annu Rev Psychol 48:649-684

Feldman RS, Meyer J, Quenzer LF (1997) Principles of neuropsychopharmacology. Sinauer Associates, Sunderland

Ferrea S, Winterer G (2009) Neuroprotective and neurotoxic effects of nicotine. Pharmacopsychiatry 42(6):255-265

Field A (2009) Discovering statistics using SPSS. Sage, London

Finke K, Bublak P, Krummenacher J, Kyllingsbæk S, Muller HJ, Schneider WX (2005) Usability of a theory of visual attention (TVA) for parameter-based measurement of attention I: evidence from normal subjects. J Int Neuropsychol Soc 11(7):832-842

Finke K, Dodds CM, Bublak P, Regenthal R, Baumann F, Manly T et al (2010) Effects of modafinil and methylphenidate on visual attention capacity: a TVA-based study. Psychopharmacology 210 (3):317-329

Foulds J, Stapleton J, Swettenham J, Bell N, McSorley K, Russell MA (1996) Cognitive performance effects of subcutaneous nicotine in smokers and never-smokers. Psychopharmacology 127(1):31-38

Green DM, Swets JA (1966) Signal detection theory and psychophysics. Wiley, New York

Greenwood PM, Fossella J, Parasuraman R (2005) Specificity of the effect of a nicotinic receptor polymorphism on individual differences in visuospatial attention. J Cogn Neurosci 17 (10): $1611-1620$

Habekost T, Rostrup E (2006) Persisting asymmetries of vision after right side lesions. Neuropsychologia 44(6):876-985

Habekost T, Rostrup E (2007) Visual attention capacity after right hemisphere lesions. Neuropsychologia 45(7):1474-1488

Hahn B, Ross TJ, Yang Y, Kim I, Huestis MA, Stein EA (2007) Nicotine enhances visuospatial attention by deactivating areas of the resting brain default network. J Neurosci 27(13):34773489

Hahn B, Ross TJ, Wolkenberg FA, Shakleya DM, Huestis MA, Stein EA (2009) Performance effects of nicotine during selective attention, divided attention, and simple stimulus detection: an fMRI study. Cereb Cortex 19(9):1990-2000

Heishman SJ, Henningfield JE (2000) Tolerance to repeated nicotine administration on performance, subjective, and physiological responses in nonsmokers. Psychopharmacology 152 (3):321-333

Heishman SJ, Taylor RC, Henningfield JE (1994) Nicotine and smoking: a review of effects on human performance. Exp Clin Psychopharmacol 2(4):345-395

Heishman SJ, Kleykamp BA, Singleton EG (2010) Meta-analysis of the acute effects of nicotine and smoking on human performance. Psychopharmacology 210(4):453-469

Jensen CG, Vangkilde S, Frøkiær V, Hasselbalch SG (2011) MBSR affects attention - or is it attentional effort? A randomized controlled trial (in press)

Jones GMM, Sahakian BJ, Levy R, Warburton DM, Gray JA (1992) Effects of acute subcutaneous nicotine on attention, information processing and short-term memory in alzheimer's disease. Psychopharmacology 108(4):485-494

Kleykamp BA, Jennings JM, Blank MD, Eissenberg T (2005) The effects of nicotine on attention and working memory in neversmokers. Psychol Addict Behav 19(4):433-438

Kyllingsbæk S (2006) Modeling visual attention. Behav Res Meth 38 (1):123-133 
Lawrence NS, Ross TJ, Stein EA (2002) Cognitive mechanisms of nicotine on visual attention. Neuron 36(3):539-548

Levin ED, McClernon FJ, Rezvani AH (2006) Nicotinic effects on cognitive function: behavioral characterization, pharmacological specification, and anatomic localization. Psychopharmacology 184(3-4):523-539

Meinke A, Thiel CM, Fink GR (2006) Effects of nicotine on visuospatial selective attention as indexed by event-related potentials. Neuroscience 141(1):201-212

Mentis MJ, Sunderland T, Lai J, Connolly C, Krasuski J, Levine B et al (2001) Muscarinic versus nicotinic modulation of a visual task. A PET study using drug probes. Neuropsychopharmacology 25 (4):555-564

Metherate R (2004) Nicotinic acetylcholine receptors in sensory cortex. Learn Mem 11(1):50-59

Myers CS, Taylor RC, Moolchan ET, Heishman SJ (2008) Doserelated enhancement of mood and cognition in smokers administered nicotine nasal spray. Neuropsychopharmacology 33 (3):588-598

Newhouse PA, Potter A, Singh A (2004) Effects of nicotinic stimulation on cognitive performance. Curr Opin Pharmacol 4 (1):36-46

Oldfield R (1971) The assessment and analysis of handedness: the Edinburgh inventory. Neuropsychologia 9:97-113

Olivers CNL, Nieuwenhuis S (2006) The beneficial effects of additional task load, positive affect, and instruction on the attentional blink. J Exp Psychol Hum Percept Perform 32 (2):364-379

Parasuraman R, Greenwood PM, Kumar R, Fossella J (2005) Beyond heritability. Psychol Sci 16(3):200-207

Petersen SE, Robinson DL, Morris JD (1987) Contributions of the pulvinar to visual spatial attention. Neuropsychologia 25 (1):97-105

Phillips JM, McAlonan K, Robb WG, Brown VJ (2000) Cholinergic neurotransmission influences covert orientation of visuospatial attention in the rat. Psychopharmacology 150(1):112-116

Posner MI, Snyder CRR, Davidson BJ (1980) Attention and the detection of signals. J Exp Psychol Gen 109(2):160-174

Redel P, Bublak P, Sorg C, Kurz A, Förstl H, Müller HJ et al. (2011) Deficits of spatial and task-related attentional selection in mild cognitive impairment and Alzheimer's disease. Neurobiol Aging. doi:10.1016/j.neurobiolaging.2010.05.014

Rezvani AH, Bushnell PJ, Levin ED (2002) Effects of nicotine and mecamylamine on choice accuracy in an operant visual signal detection task in female rats. Psychopharmacology 164 (4):369-375

Rose EJ, Ross TJ, Kurup PK, Stein EA (2010) Nicotine modulation of information processing is not limited to input (attention) but extends to output (intention). Psychopharmacology 209(4):291-302

Rusted JM, Trawley S (2006) Comparable effects of nicotine in smokers and nonsmokers on a prospective memory task. Neuropsychopharmacology 31(7):1545-1549

Rusted JM, Warburton DM (1992) Facilitation of memory by posttrial administration of nicotine: evidence for an attentional explanation. Psychopharmacology 108(4):452-455

Rusted JM, Sawyer R, Jones C, Trawley SL, Marchant NL (2009) Positive effects of nicotine on cognition: the deployment of attention for prospective memory. Psychopharmacology 202(13):93-102

Rycroft N, Rusted JM, Hutton SB (2005) Acute effects of nicotine on visual search tasks in young adult smokers. Psychopharmacology 181(1):160-169

Sarter M, Parikh V (2005) Choline transporters, cholinergic transmission and cognition. Nat Rev Neurosci 6(1):48-56

Sarter M, Bruno JP, Givens B (2003) Attentional functions of cortical cholinergic inputs: What does it mean for learning and memory? Neurobiol Learn Mem 80(3):245-256

Sarter M, Hasselmo ME, Bruno JP, Givens B (2005) Unraveling the attentional functions of cortical cholinergic inputs: interactions between signal-driven and cognitive modulation of signal detection. Brain Res Rev 48(1):98-111

Sherwood N (1993) Effects of nicotine on human psychomotor performance. Human Psychopharmacol 8(3):155-184

Shibuya H, Bundesen C (1988) Visual selection from multielement displays: measuring and modeling effects of exposure duration. $\mathrm{J}$ Exp Psychol Hum Percept Perform 14(4):591-600

Sperling G (1960) The information available in brief visual presentations. Psychol Monog Gen Appl 74(11):1-29

Spurden DP, Court JA, Lloyd S, Oakley A, Perry R, Pearson C et al (1997) Nicotinic receptor distribution in the human thalamus: autoradiographical localization of $[3 \mathrm{H}]$ nicotine and [125I] [alpha]-bungarotoxin binding. J Chem Neuroanat 13(2):105-113

Stough C, Mangan G, Bates T, Frank N, Kerkin B, Pellett O (1995) Effects of nicotine on perceptual speed. Psychopharmacology 119(3):305-310

Thiel CM, Fink GR (2008) Effects of the cholinergic agonist nicotine on reorienting of visual spatial attention and top-down attentional control. Neuroscience 152(2):381-390

Thiel CM, Zilles K, Fink GR (2005) Nicotine modulates reorienting of visuospatial attention and neural activity in human parietal cortex. Neuropsychopharmacology 30(4):810-820

Thompson JC, Stough C, Ames D, Ritchie C, Nathan PJ (2000) Effects of the nicotinic antagonist mecamylamine on inspection time. Psychopharmacology 150(1):117-119

Thompson JC, Wilby G, Stough C (2002) The effects of transdermal nicotine on inspection time. Hum Psychopharmacol 17(3):157-161

Vossel S, Thiel CM, Fink GR (2008) Behavioral and neural effects of nicotine on visuospatial attentional reorienting in non-smoking subjects. Neuropsychopharmacology 33(4):731-738

Vossel S, Warbrick T, Mobascher A, Winterer G, Fink GR (2011) Spatial and sustained attention in relation to smoking status: behavioural performance and brain activation patterns. J Psychopharmacol (in press)

Wesnes K, Warburton DM, Matz B (1983) Effects of nicotine on stimulus sensitivity and response bias in a visual vigilance task. Neuropsychobiology 9(1):41-44

Witte EA, Davidson MC, Marrocco RT (1997) Effects of altering brain cholinergic activity on covert orienting of attention: comparison of monkey and human performance. Psychopharmacology 132(4):324-334

Yerkes RM, Dodson JD (1908) The relation of strength of stimulus to rapidity of habit-formation. J Comp Neurol Psychol 18(5):459482

Yu AJ, Dayan P (2005) Uncertainty, neuromodulation, and attention. Neuron 46(4):681-692 